\title{
Management of the risk of bronchoaspiration in patients with oropharyngeal dysphagia
}

\author{
Layanne Ferreira dos Santos Carmo ${ }^{(1)}$ \\ Fabíola Andrea Andrade dos Santos ${ }^{(1)}$ \\ Simonize Cunha Barreto de Mendonça ${ }^{(1)}$ \\ Brenda Carla Lima Araújo(2)
}

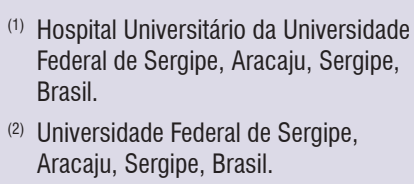

Conflict of interests: Nonexistent

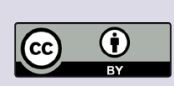

Received on: April 17, 2018 Approved on: July 4, 2018

Corresponding author: Layanne Ferreira dos Santos Carmo Rua João de Oliveira, 120, Centro CEP: 49200-000 - Estância, Sergipe, Pernambuco, Brasil

E-mail: layanne.gr@gmail.com

\section{ABSTRACT}

Objective: to manage the risk of bronchoaspiration in patients with oropharyngeal dysphagia through signalizing plates in the hospital bed.

Methods: a descriptive, quantitative study, developed in the medical clinic I (diagnostic investigation), medical clinic II (infectology / pneumology), surgical clinics and intensive care unit of the University Hospital of the Federal University of Sergipe, from February to August 2017. It was composed of patients in the age group $\geq 18$ years of age, of both sexes, diagnosed with oropharyngeal dysphagia, based on the application of the adapted protocol of clinical evaluation in the hospital bed and instrumental examination of swallowing, when pertinent. Following the clinical evaluation and identification of the risk of bronchoaspiration due to oropharyngeal dysphagia, the signs were inserted at the hospital bed by the speech therapist.

Results: the study included 43 patients at risk of bronchoaspiration due to oropharyngeal dysphagia, average age of $53.7 \pm 3.53$ years old, with $51.1 \%(n=22)$ females and $48.9 \%(n=21)$ males. A predominance of neuropathies $(53.4 \%)$ and severe neurogenic oropharyngeal dysphagia (37.2\%) was observed. The intensive care unit stood out with $44.18 \%(n=19)$ of the application of signs of risk of bronchoaspiration.

Conclusion: the management of bronchoaspiration risk has been shown to be a promising measure to reduce adverse events, which affect the patient's safety and consequently the quality of care in the hospital environment, as well as a possible strategy for measuring respiratory complications triggered by aspiration of oropharyngeal contents.

Keywords: Deglutition Disorders; Speech, Language and Hearing Sciences; Pneumonia, Aspiration 


\section{INTRODUCTION}

Bronchoaspiration is considered one of the main indicators of dysphagia and the most worrying ${ }^{1,2}$. It occurs through the infiltration of food particles, oropharyngeal fluids or gastric contents into the lower airways, which can trigger infectious pneumonia, chemical pneumonitis and respiratory distress syndrome ${ }^{1}$. These complications contribute to a significant increase in morbidity and mortality rates, prolonging, on average, 5 to 9 days the hospitalization time of patients and significantly increasing hospital costs ${ }^{3}$.

Hospital pneumonia is one of the major causes of death from hospital-acquired infections ${ }^{4}$. In the United States, it is listed as the second most frequently found hospital infection in adults and the annual costs per infected patient exceed $\$ 13,932.00^{5,6}$. In Brazil, it is the fourth leading cause of hospitalization, with the elderly being more affected, due to the increased incidence of dysphagia and gastroesophageal reflux in this age group ${ }^{7}$.

In nursing homes, the prevalence of pneumonia ranges from $13 \%$ to $48 \%$ among all infections. Approximately $70 \%$ of aspiration rates correspond to patients with lowering of consciousness level, $50 \%$ in dysphagic patients after stroke and $50 \%$ to $75 \%$ in patients with laryngotracheal intubation ${ }^{8}$.

In addition to neurological diseases and orotracheal intubation, other factors are associated with the risk of bronchoaspiration, highlighting the use of enteral diets, psychiatric and central nervous system depressants, head and neck surgeries, neoplasms, pulmonary diseases and the process of aging 9 .

The occurrence of aspiration may trigger a number of clinical signs, including resting tachypnea, bilateral wheezing in the pulmonary auscultation and reduction of arterial oxygenation. However, some episodes of aspiration do not bring about perceptible changes during clinical evaluation, which is called silent aspiration ${ }^{10}$.

In order to minimize the aggravating factors inherent in patients at risk of aspiration, some interventions were recommended by the Nursing Intervention Classification (NIC) ${ }^{11}$, such as: positioning the patient vertically with an angle equal to or greater than 30 , being able to reach up to 90 o if possible; monitoring/ the level of consciousness and pulmonary status; evaluating the cough reflex, swallowing ability; vomiting control; keeping the head elevated 30 to 40 minutes after feeding; inspecting for food or medicine held in the oral cavity; providing mouth care and checking the gastric / enteral / gastrostomy probes.

Given this perspective, the Brazilian Ministry of Health establishes the Programa Nacional de Segurança do Paciente (National Patient Safety Program) through the ordinance $529 / 2013$, considering the need for risk management based on the establishment of strategies, products and actions that allow managers and health professionals to attenuate adverse events regarding healthcare, including care-related infections ${ }^{12}$.

Therefore, prevention is the main objective in the care of the patient with risk of aspiration ${ }^{13}$, however, few national studies address the hospital practice and the relevance of actions aimed at prevention of bronchoaspiration. Based on this, the present study aimed to manage the risk of bronchoaspiration in patients with oropharyngeal dysphagia through signposts, due to the need to incorporate the management of this risk in health care and, thus, contribute to the reduction of clinical and respiratory complications.

\section{METHODS}

This study was approved by the Ethics and Research Committee of the Federal University of Sergipe under the pronouncement No. 1,275,790 in October 2015 and submitted to the evaluation of the Serviço de Controle de Infecção Relacionada à Assistência à Saúde (Service of Control of Infection Related to Health Care) - SCIRAS - for applicability in the institution.

This is a descriptive, cross-sectional, quantitative study that aimed at managing the risk of bronchoaspiration in patients with oropharyngeal dysphagia (mechanical and/or neurogenic) through signaling plates in hospital bed. Belonging to a larger project entitled "Caracterização da disfagia orofaríngea dos pacientes internados no Hospital", developed at the University Hospital of Sergipe by a resident of the Programa Saúde do Adulto e do Idoso da Residência Multiprofissional em Saúde (Adult and Elderly Health Program of the Multiprofessional Health Residency), from February to August 2017.

It covered all patients of both sexes, aged $\geq 18$ years old, hospitalized in the medical clinic I (diagnostic investigation), medical clinic II (pneumology and infectology), surgical clinic and intensive care unit (ICU).

The inclusion criteria were: to present a risk of bronchoaspiration due to oropharyngeal dysphagia, indicated through Speech, Language and Hearing Sciences clinical evaluation and to be hospitalized in the wards of Medical Clinics I and II, Surgical Clinic 
and ICU. In addition, the exclusion criterion: to do not present a diagnosis of oropharyngeal dysphagia.

After the approval of the applicability by the SCIRAS, the proposal was presented to the speech therapists and nurses (residents and preceptors) working in the hospital wards and ICUs, so that, they would understand the objective and the criteria for the use of signs of risk of bronchoaspiration in patients with oropharyngeal dysphagia.

The selection of the patients, as shown in the flow diagram (Figure 1), occurred, initially, through adapted Speech Language Pathology screening ${ }^{14}$, applied by speech therapists. This instrument included objective questions about the functions of swallowing and chewing, with an evaluation criterion: "approval" (no suggestive response of alteration in these functions) or "failure" (presence of responses indicating changes in swallowing and / or chewing). The patients who failed during the application of the screening were submitted to clinical evaluation in the hospital bed, using an evaluation protocol adapted from Padovani et al. $(2007)^{15}$, subdivided into structural and functional evaluation of swallowing.

The protocol comprehends the following aspects: patient identification, vital parameters, level of consciousness, communication aspects, consistency of current feeding and supply mode (when on an oral diet), tone classification, mobility and sensitivity (hypo-sensitivity, hypersensitivity and adequate), vocal aspects, teeth condition, characteristics of saliva and food swallowing (liquid, semi-liquid, pasty and solid). Next, were performed the classification of dysphagia regarding type: neurogenic and mechanical; and degree: mild, mild to moderate, moderate to severe and severe. Therefore, the conduct was defined: speech therapy, swallowing management and guidelines. Lastly, the definition of the functional level of oral intake was based on the Functional Oral Intake Scale-FOIS ${ }^{16}$. In peculiar cases (suspected silent aspiration), a complementary instrumental evaluation was requested through functional endoscopic evaluation of swallowing (FEES).

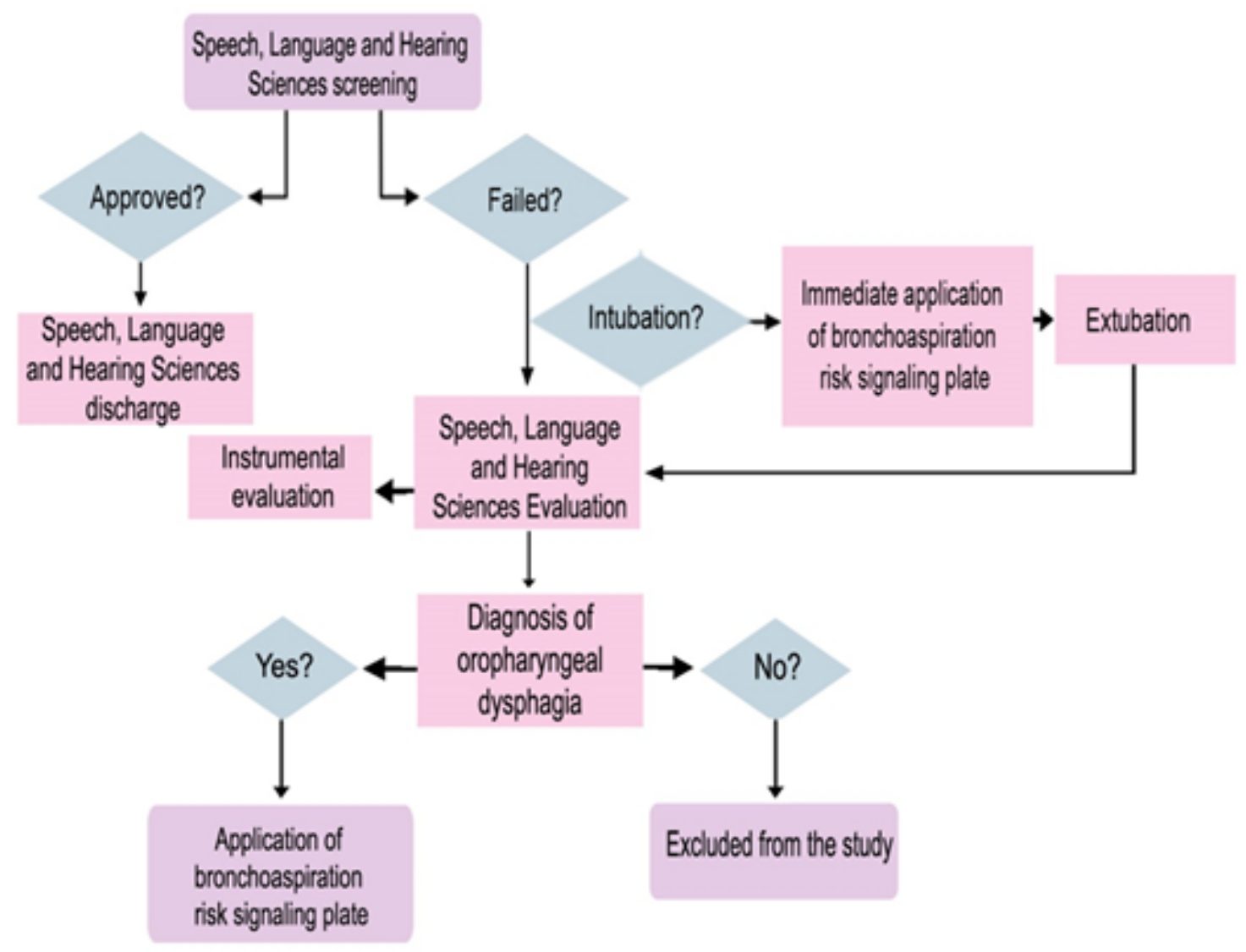

Figure 1. Patient selection process flowchart 
The collection of the variables age, sex and clinical diagnosis of the participants was based on the consultation of the respective medical records available in the wards. The description of the type, degree of oropharyngeal dysphagia and the speech therapy conduct all followed the completion of the clinical evaluation protocol in the hospital bed.

Subsequent to the evaluation and definition of the Speech, Language and Hearing Sciences diagnosis of oropharyngeal dysphagia, the signs indicating the risk of bronchoaspiration, identified by the pink color, with dimensions $22 \mathrm{~cm} \times 4 \mathrm{~cm}$ (Figure 2), were inserted next to the other pre-existing ones in the hospital. They indicated risk of falls, precautions of contact, risk of pressure ulcer and medication restriction, and were positioned on the wall, in medial and superior positions to the headboard of the hospital bed.

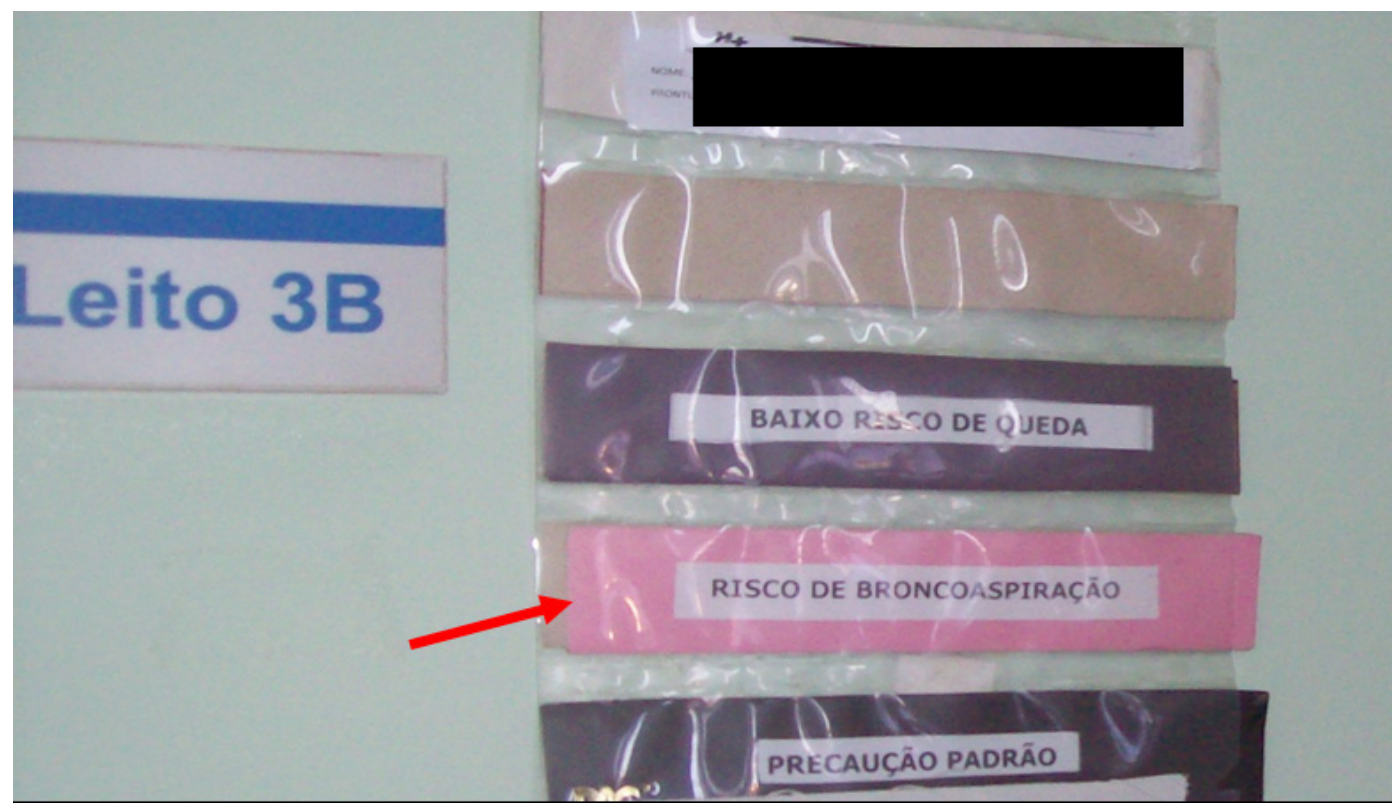

Legend: Leito 3B = Bed 3B

Baixo risco de queda $=$ Low fall risk

Risco de broncoaspiração = Bronchoaspiration risk

Precaução padrão = standard precaution

Figure 2. Disposition of the signal board of the bronchoaspiration risk in the hospital bed

In cases of contraindication to direct Speech, Language and Hearing Sciences assessment, such as in orotracheal intubation, commonly found in patients in the ICU, the signaling plates were inserted immediately after the screening. This happened since the risk of bronchoaspiration was imminent in these situations, due to possible dysphagia caused by the presence of the orotracheal tube, which makes the adequate swallowing dynamics unfeasible, even in a transitory way ${ }^{17,18}$. In these circumstances, the speech therapy monitoring was indirectly indicated, that is, the patients were monitored by the speech therapy team until the time of extubation, when they was submitted to swallowing assessment and subsequent interventions, after a joint deliberation with a medical staff.
It is noteworthy that, following the application of signs of bronchoaspiration risk, preventive measures against aspiration were established, such as: adequacy of patient positioning in the hospital bed, guidelines for oral diet administration for patients in this clinical condition (mode, volume, rhythm, consistency and supply tools) and speech therapy.

In contrast, the use of bronchial aspiration risk signaling was also susceptible to discontinuation in any study patients, when the risk of bronchoaspiration due to oropharyngeal dysphagia was suppressed.

The analysis of the data was performed through simple calculations in percentages using the Excel ${ }^{\circledR}$ software and exposed in a descriptive way, using tables and figures. 


\section{RESULTS}

The present study included 43 participants at risk of bronchoaspiration, due to oropharyngeal dysphagia, with an average age of $53.7 \pm 3.53$ years old, of which, $22(51.1 \%)$ were female and 21 (48.9\%) were male. The swallowing assessment in the hospital bed revealed 26 (60.42\%) cases of neurogenic oropharyngeal dysphagia of the neurogenic type and 17 (39.47\%) cases of mechanical oropharyngeal dysphagia. Regarding the severity of dysphagia, a predominance of patients with severe oropharyngeal dysphagia (Figure 3) and predominance of neuropathies (53.4\%) were observed, among which are: stroke, neurotoxoplasmosis and Parkinson's disease. These were the most found according to data collection in medical records.

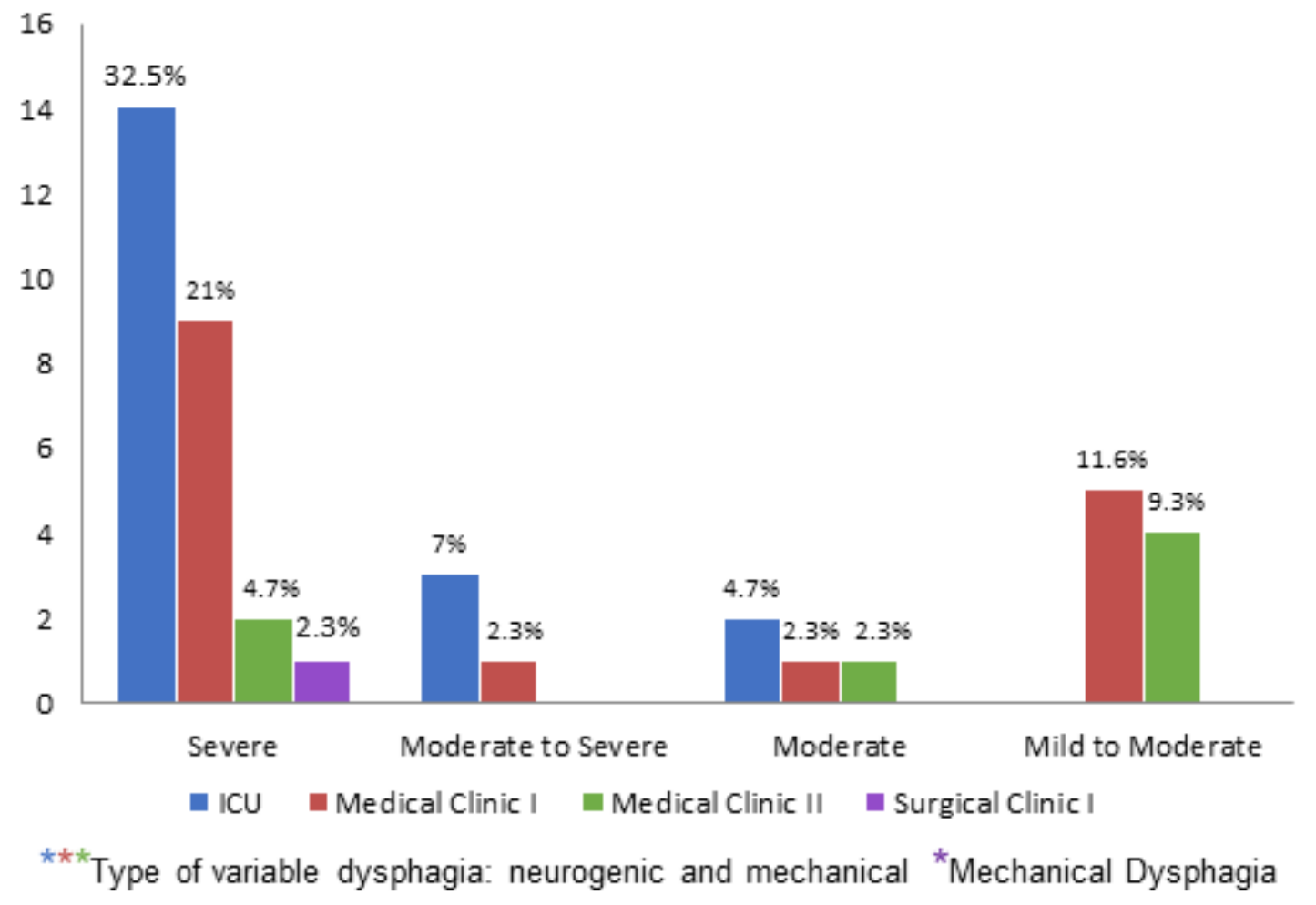

Figure 3. Type and severity of oropharyngeal dysphagia of study participants by hospitalization sector

The signs signalizing the risk of bronchoaspiration were arranged close to the hospital beds of all the infirmaries and ICU, as described in the table below:

Based on Table 1, the ICU presented a higher incidence of risks for bronchoaspiration, also occupying a predominant position regarding the severity of dysphagia, followed by Medical Clinic I. On the other hand, the Surgical Clinic was the sector with less application demands.

Table 1. Distribution of signaling boards of bronchoaspiration risk by hospitalization sector

\begin{tabular}{ccc}
\hline Hospital Wards & \multicolumn{3}{c}{ Signaling Plates } \\
\cline { 2 - 3 } & No & $\mathbf{n} \%$ \\
\hline Medical Clinic I & 16 & $37.2 \%$ \\
Medical Clinic II & 7 & $16.2 \%$ \\
Surgical Clinic I & 1 & $2.32 \%$ \\
Intensive Care Unit & 19 & $44.18 \%$ \\
\hline Total & 43 & $100 \%$ \\
\hline
\end{tabular}


Regarding the adhesion of the proposed use of signaling plates, the professionals involved - eight residents and three preceptors, totaling eleven speech therapists - unanimously adhered to the use, but failed episodes of risk management were observed, for example, the absence of the signs of risk of bronchoaspiration in situations of necessary applicability, as well as their permanence when there was no risk of bronchoaspiration due to oropharyngeal dysphagia.

The layout of the signs of bronchoaspiration risk occurred in a simple and provisional way, following the model of the pre-existing plaques in the institution.

\section{DISCUSSION}

The risk of bronchoaspiration is manifested with high prevalence in large hospitals ${ }^{19}$ and may be associated with events of pneumonitis or aspiration pneumonia, which is considered the most common infection in the hospital environment, despite its occurrence in the community. In this context, literature, especially in the international domain, suggests prevention and declares it as the main goal in the care of patients with such risk ${ }^{20}$. With this objective in mind, health risk management has been frequently cited and implemented in hospital organizations, in order to minimize undesirable or unexpected events, from the conducts, procedures and resources that detect, classify and monitor the risks inherent in health care ${ }^{21}$.

However, it is essential to know the profile and clinical characteristics of the patients to implement the measures of prevention and control of pneumonia ${ }^{20}$. The analysis of the following variables gender, type and degree of dysphagia indicated a predominance of women, corroborating with the literature that states a higher incidence of underlying diseases and comorbidities in females ${ }^{19}$, besides the increased risk of dysphagia in this population ${ }^{21}$.

Results of a study carried out at the Brasília University Hospital, which aimed to investigate the association between the degree of risk of dysphagia and the nutritional risk of hospitalized elderly patients, concluded that, in males, the difference was reduced between minimum risk (34\%) and greater risk (66\%) for dysphagia, while in females, this difference was high, with a minimal risk of $25 \%$ and higher risk of $75 \%^{22}$.

Regarding the type of dysphagia, there was a predominance of neuropathic patients (neurogenic dysphagia) submitted to the use of signs regarding the risk of bronchoaspiration. This result is due to the fact that the symptoms present in neurological diseases, such as paresis, paralysis and reduced contraction of the pharynx minimize the protection of the lower airways ${ }^{23}$, resulting in a high incidence of bronchoaspiration in patients with neuropathies, followed by diabetic and cardiopathy patients, with statistically significant differences between them ${ }^{19}$.

Studies of the elderly population, mainly with victims of stroke, associated the presence of typical dysphagia of neurological diseases with the risk of aspiration pneumonia, representing $5 \%$ to $15 \%$ of the diagnoses of pneumonia in hospitalized patients. In addition, the administration of sedative and antipsychotic drugs interfere with the mechanism of swallowing and, therefore, may increase the risk of aspiration ${ }^{10,24}$.

Another study carried out in Germany, at a hospital center in the city of Herne, covering 209 hospitalized patients with aspiration pneumonia, aiming to establish valid prognostic tools for this group, pointed out the presence of dysphagia in almost two thirds of their patients, due to central nervous system disorders, such as stroke, dementia and Parkinson's disease ${ }^{25}$.

Aspiration pneumonia is a serious disease with a diagnosis that is difficult to distinguish between other kinds of pneumonia, and, therefore, it is underdiagnosed. The investigation encompasses clinical history, signs, symptoms, laboratory tests and chest radiography. Treatment includes antibiotics, which raises hospital financial costs and prolong the hospitalization time $^{10}$.

A research regarding the budgetary impact, in a tertiary-level hospital involving 66 patients, with the objective of estimating the prevalence of bronchoaspiration and verifying the cost-effectiveness of the application of a preventive protocol was performed. This research states that an institution of 100 hospital beds and a percentage of $56.06 \%$ patients with risk of aspiration and indication of Speech, Language and Hearing Sciences assessment presented an estimated cost of $R \$ 42,045.00$, per month, considering the amount of $R \$ 100.00$ for each speech therapy session and the 4-day in-hospital stay time ${ }^{19}$. These authors concluded that the adherence of a preventive program through the application of a protocol, including the speech therapist, is cost-effective, generating a gain of $\mathrm{R} \$ 900.00$ per hospitalization and a reduction of 0.15 days of hospitalization per patient.

Several other proposals are disseminated in the literature, one is the Guidelines for preventing Healthcareassociated Pneumonia issued by the Center for Disease Control and Prevention, which describes the following 
as practices of prevention of pneumonia: education of health professionals on epidemiology and infection control procedures, implementation of interventions to prevent pneumonia and monitoring of pneumonia ${ }^{26}$.

A descriptive, qualitative study carried out in a medium-sized hospital with the objective of identifying the nursing team's conception of the possible risks to the patient's safety in the clinical unit, as well as the strategies used to control the risk, concluded that professionals identify the risks inherent in health care and intervene through standardized preventive behaviors. Among these, the insertion of bracelets in hospitalized patients, with specific colors for each level of risk, was applied after evaluation. This is recorded in an information system and attached to the patient's medical record by the nursing team, in hospital admission units ${ }^{27}$.

In the present study, the ICU assumed the position of predominance among the other sectors, regarding the greater urgency in the use of signs indicating the risk of bronchoaspiration, corroborating with the scientific findings, which indicate a high risk of bronchoaspiration and aspiration pneumonia in patients under intensive care ${ }^{19,28}$. This result may also justify the prevalence of severe dysphagia in the patients of the present study, since these presented aggravating multi-factors for the risk of bronchoaspiration ${ }^{29}$, such as the underlying disease itself and the presence of orotracheal tube.

A retrospective research, including patients over the age of 18 years old, conducted at the University Hospital in Salt Lake City, Utah, USA, 520 hospital beds, aimed at identifying a population of patients with aspiration pneumonia, stated that patients with this condition are common in the ICUs, and they present a higher rate of comorbidities and mortality. In the outpatient setting, 177 of these patients were submitted to swallowing evaluation and dysphagia was present in $96 \%$ of them? ${ }^{7}$.

Altman, Yu and Schaefer $(2010)^{30}$ compared the occurrence of diseases among hospitalized patients with and without dysphagia and concluded that cases with dysphagia were significantly associated with aspiration pneumonia, as well as the high risk of mortality in dysphagic patients when compared to patients without dysphagia. The difference was also significant in the length of hospital stay, with a $40 \%$ increase in hospitalization time in patients diagnosed with dysphagia in all age groups.
For these authors, it is recommended for patients at risk of aspiration to be identified early. Their assistance should include a plan of care encompassing evaluation, appropriate intervention and aspiration precautions, especially with patients in critical conditions: advanced age, pneumonia, heart diseases, dehydration, malnutrition, and neuropathies, such as stroke and neurodegenerative diseases ${ }^{30}$.

Sousa ${ }^{13}$, in his systematic review study, containing 16 randomized controlled trials on effective strategies / interventions for the prevention of aspiration pneumonia in adults, including oral hygiene, aromatherapy, diet management and compensatory positions, states that neurosurgical patients have a greater risk of developing pneumonia when compared to patients undergoing laparotomy or other procedures.

Therefore, it is possible to justify the low use of signs of bronchoaspiration risk in the surgical clinics, since these wards do not work with neurosurgery patients. Nevertheless, the team must maintain the management of the risk of bronchoaspiration in these sectors, due to the post-surgical digestive and respiratory changes, as well as a state of consciousness depressed by general anesthesia, which are considered risk factors for aspiration ${ }^{31}$.

The multidisciplinary team should monitor the signs and diagnoses of dysphagia, so that, aspiration precautions can be implemented immediately ${ }^{32}$. In the study in question, some failed episodes were observed in the management of the risk of bronchoaspiration, as absence of signaling plates when needed, or presence of it when the risk was nonexistent. This fragility in teamwork affects the safety culture of the patient, increasing the risk of complications and deaths among surgical patients, as well as interfere with reliability, which is defended as the basis of quality care ${ }^{33}$.

Lastly, it is worth highlighting some limitations of this study, such as the vulnerability in monitoring the use of signs indicating the risk of bronchoaspiration, caused by the unviability of the researcher to go through all the infirmaries regularly and the lack of systematic training of the professionals involved in the process. Therefore, the reduced explanation about the present theme in the national literature, specially focused on the Speech, Language and Hearing Sciences practice in the hospital, restricted the theoretical framework. Thus, the need for further studies is noteworthy, as well as the elaboration of a bronchoaspiration risk protocol that allows greater control and visibility of risks by all multidisciplinary teams. 


\section{CONCLUSION}

The present study, aiming at managing the risk of bronchoaspiration in patients with dysphagia through signaling plates in the hospital bed, has been shown to be a promising measure to reduce adverse events, which affect the patient's safety and, consequently, the quality of care in the hospital environment, as well as a possible strategy for measuring the respiratory complications triggered by aspiration of oropharyngeal contents.

\section{REFERENCES}

1. Logemann JA. Evaluation and treatment of swallowing disorders. 2th ed. Austin, TX: Pro-ed; 1998.

2. Lefton-greif MA, Mcgrath-morrow SA. Deglutition and respiration: development, coordination, and practical implications. Seminars in speech and language. 2007;28(3):166-79. DOI: 10.1055/s-2007-984723.

3. Beck-Schimmer B, Bonvini JM. Bronchoaspiration: incidence, consequences and management. Eur J Anaesthesiol. 2011;28(2):78-84. DOI: 10.1097/ EJA.0b013e32834205a8.

4. Raghavendran K, Mylotte JM, Scannapieco FA. Nursing home-associated pneumonia, hospital-acquired pneumonia and ventilatorassociated pneumonia: the contribution of dental biofilms and periodontal inflammation. Periodontology 2000. 2007;44(1):164-77. DOI: 10.1111/j.1600-0757.2006.00206.x.

5. Swaminathan A. Aspiration pneumonia. Medscape reference. 2013. Disponivel em: http://emedicine. medscape.com/article/296198-overview\#aw2aa

6. Fuller RL, McCullough EC, Bao MZ, Averill RF. Estimating the costs of potentially preventable hospital acquired complications. Health Care Financ Rev. 2009;30(4):17-32.

7. Lanspa MJ, Jones BE, Brown SM, Dean NC. Mortality, morbidity, and disease severity of patients with aspiration pneumonia. J Hosp Med. 2013;8(2):83-90. DOI: https://doi.org/10.1002/ jhm.1996.

8. Benatti FG, Montenegro FLB. A Intervenção odontológica colaborando na diminuição das afecções respiratórias dos idosos. REV EAP. APCD. 2008;9(2):1-4.
9. DeLegge $\mathrm{MH}$. Aspiration pneumonia: incidence, mortality and at-risk populations. JPEN J Parenter Enteral Nutr. 2002;26(6):19-25.

10. DiBardino DM, Wunderink RG. Aspiration pneumonia: a review of modern trends. J Crit Care. 2015;30(1):40-8. DOI: https://doi.org/10.1016/j. jcrc.2014.07.011.

11. Bulechek GM, Butcher HK, Dochterman JM, Wagner C. Nursing Interventions Classification (NIC). 6. ed. Rio de Janeiro: Elsevier; 2016.

12. Ministério da Saúde (BR). Portaria no 529, de 1 o de abril de 2013. Institui o Programa Nacional de Segurança do Paciente (PNSP). Brasília: Ministério da Saúde; 2013.

13. Sousa NM. Prevenção da pneumonia por aspiração: revisão sistemática [Dissertação]. Porto (Portugal): Universidade do Porto; 2013.

14. Belafsky PC, Mouadeb DA, Rees CJ, Pryor JC, Postma GN, Leonard RJ et al. Validity and reliability of the Eating Assessment Tool (EAT-10). Ann Otol Rhinol Laryngol. 2008;117(2):919-24. DOI: $10.1177 / 000348940811701210$

15. Padovani AR, Moraes DP, Mangili LD, Andrade CRF. Protocolo fonoaudiológico de avaliação do risco para disfagia (PARD). Rev. soc. bras. fonoaudiol. 2007;12(3):199-205.

16. Crary MA, Mann GD, Groher ME. Initial psychometric assessment of a functional oral intake scale for dysphagia in stroke patients. Arch Phys Med Rehab. 2005;86(8):1516-20.

17. Noordally SO, Sohawon S, Gieter M, Bellout $H$, Verougstraete $G$. A study to determine the correlation between clinical, fiber-optic endoscopic evaluation of swallowing and videofluoroscopic evaluations of swallowing after prolonged intubation. Nutr Clin Pract. 2011;26(4):457-62.

18. Mota LAA, Carvalho GB, Bri VA. Laryngeal complications by orotracheal intubation: literature review. Int. Arch. Otorhinolaryngol. 2012;16(2):236-45.

19. Almeida AEM, Alcântara ACC, Lima FAM, Rocha HAL, Cremonin Junior JR, Costa HJM. Prevalência de risco moderado e alto de aspiração em pacientes hospitalizados e custo-efetividade da aplicação de protocolo preventivo. J Bras Econ Saúde. 2016;8(3):216-20.

20. Souza AS. Prevalência de pneumonia associada à assistência à saúde em unidades de terapia intensiva [Dissertação]. Campo Grande (MS): 
Universidade Federal de Mato Grosso do Sul UFMS; 2012.

21. Helidéa OL, Elisabete CRD. O gerenciamento de riscos na saúde - aplicação na atenção hospitalar. RAHIS. 2010;5(2):89-90 DOI: http://dx.doi. org/10.21450/rahis.v0i5

22. Maciel JRV, Oliveira CJR, Tada CMP. Associação entre risco de disfagia e risco nutricional em idosos internados em hospital universitário de Brasília. Rev. Nutr. 2008;(21)4:411-21. DOI: 10.1590/ S1415-52732008000400005.

23. Macedo EDMF. Estudo comparativo entre a videoendoscopia da deglutição e a videofluoroscopia para a avaliação de disfagia orofaríngea de causa neurogênica [Tese]. Curitiba (PR): Universidade Federal do Paraná; 2001.

24. Knol W, VanMarum RJ, Jansen PA, Souverein PC, Schobben AF, Egberts AC. Antipsychotic drug use and risk of pneumonia in elderly people. J Am Geriatr Soc. 2008;56(4):661-6.

25. Heppner HJ, Sehlhoff B, Niklaus D, Pientka L, Thiem U. Pneumonia Severity Index (PSI), CURB-65, and mortality in hospitalized elderly patients with aspiration pneumonia. J Gerontol Geriatr. 2011;44(4):229-34. DOI: 10.1007/ s00391-011-0184-3.

26. CDC - Center for Disease Control and Prevention. Guidelines for preventing. Health Care Associated Pneumonia, 2003. MMWR Recomm Rep. 2004;53(RR-3):1-36.

27. Fassini $P$, Hahn GV. Riscos à segurança do paciente em unidade de internação hospitalar: concepções da equipe de enfermagem. Rev Enferm UFSM. 2012;2(2):290-9.

28. Toufen JC, Camargo FP, Carvalho CRR. Pneumonia aspirativa associada a alterações da deglutição: relato de caso. Rev. bras. ter. intensiva. 2007;19(1):118-22. DOI: http://dx.doi.org/10.1590/ S0103-507X2007000100016.

29. Marik PE. Aspiration pneumonitis and aspiration pneumonia. N Engl J Med. 2001;344(9):665-71.

30. Altman KW, Yu GP, Schaefer SD. Consequence of dysphagia in the hospitalized patient: impact on prognosis and hospital resources. Arch Otolaryngol Head Neck Surg. 2010;136(8):784-9. http://dx.doi. org/10.1001/archoto.2010.129.

31. Xiaowen Hu, Lee JS, Pianosi PT, Ryu JH. Aspiration-related pulmonary syndromes. CHEST. 2015;147(3):815-23.
32. Eisenstadt ES. Dysphagia and aspiration pneumonia in older adults. $J$ Am Acad Nurse Pract. 2010;22(1):17-22. DOI: 10.1111/j.1745-7599.2009.00470.x.

33. Pham JC, Aswani MS, Rosen M, Lee H, Huddle $\mathrm{M}$, Weeks $\mathrm{K}$ et al. Reducing medical errors and adverse events. Annu Rev Med. 2012;63(1):447-63. DOI: 10.1146 / annurev-med-061410-121352. 